\title{
New Recording Setup for Ratiometric Recording of Action Potentials by Optical Means
}

\author{
J Bardonova $^{1}$, I Provaznik ${ }^{1}$, M Novakova $^{2}, \mathrm{~K}_{\text {Nogova }^{2}}{ }^{\text {, J Sekora }}{ }^{1}$ \\ ${ }^{1}$ Brno University of Technology, Brno, Czech Republic \\ ${ }^{2}$ Masaryk University, Brno, Czech Republic
}

\begin{abstract}
Monophasic action potential (MAP) can be recorded from the heart surface by optical method via fluorescence measurement. The motion of isolated heart during experiment caused additional noise in recorded signal. The motion artifact can be eliminated by ratiometric fluorescence emission measurements.

This study is based on experiments in which a new ratiometric recording setup is used for recording MAPs from isolated rabbit hearts perfused according to Langendorff. MAPs recorded by new ratiometric recording setup are compared with simple optical MAP measurement without ratiometry.
\end{abstract}

\section{Introduction}

The recording of monophasic action potentials by optical method with transmembrane voltage-sensitive dyes (VSDs) is successfully used to study cardiac electrophysiological responses under various experimental conditions [1]. This method represents possibility to record electrical changes on the cardiomyocyte membrane in situations where use of classical electrodes is difficult or even impossible (e.g. instable electrical field, during defibrillation, etc.). It can be considered a valuable mean of studying electrical events in the whole range of cardiac preparations - from the isolated cardiac myocytes to the heart in situ, in healthy as well as in diseased myocardium.

In this paper, a new recording setup for ratiometric fluorescence emission measurements is presented. The proposed method for modified ratiometric measurements exploits newly available segmented narrow-band photodiodes. They consist of three sensors in a single package, each of them sensitive in non-overlapping wavelength bands. A new method is compared with simple optical MAPs measurement [2].

\section{Methods}

The methods used in the projects are based on animal experiments in which MAP is recorded from isolated rabbit heart perfused according to Langendorff. MAP was recorded by simple optical method [2] and by new proposed method. MAPs are compared by signal-to-noise ratio (SNR).

\subsection{Animal experiments}

Six isolated New Zealand rabbit hearts were perfused at Langendorff setup. Three hearts were studied using simple optical MAP measurement. In case of the other three hearts, proposed system with a segmented light sensor was used.

The isolated heart is pleased into temperature controlled bath filled with Krebs-Henseleit solution and heart coronary system is filled through aorta. The performance and viability of spontaneously beating heart is controlled during the whole experiment via recording of three electrocardiographic leads.

Each experiment consisted of five phases: isolating the heart, control period, staining with the dye, dye washout and MAPs recording. Duration of each period is approximately 20 minutes. Experimental conditions were kept stable in all experiments $\left(37^{\circ} \mathrm{C}, 1.25 \mathrm{mM} \mathrm{Ca}^{2+}\right)$.

All experiments followed the guidelines for animal treatment approved by local authorities and conformed to the EU law.

\subsection{Voltage sensitive dye}

The fluorescent voltage-sensitive dye di-4-ANEPPS (amino-naphthyl-ethenyl-pyridinium) produced by Molecular Probes, Inc. is used in our experiments. VSD's spectral characteristics are dependent on the type of tissue and solution in which it is applied. The absorption spectrum of excitation light ranges over spectrum of blue visible light. The fluorescence emission spectrum ranges over spectrum of green and red visible light and depends on surface voltage. The fluorescence emission moves from left to right with increasing of membrane potential. Corresponding spectral characteristics for application of di-4-ANEPPS VSD in rabbit heart tissue can be seen in Figure 1. 


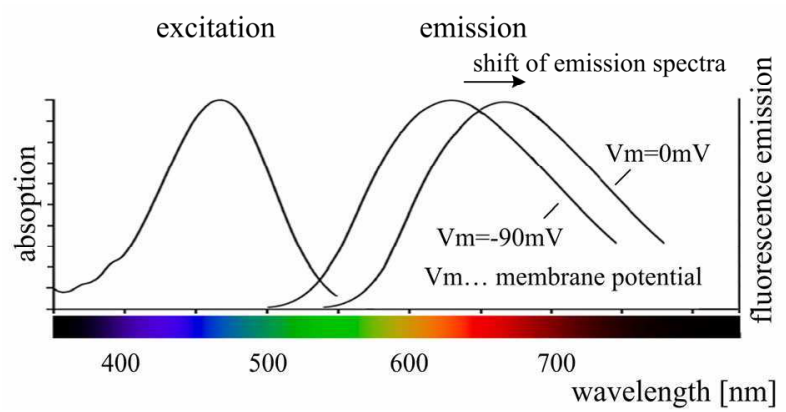

Figure 1. Spectral characteristics of voltage-sensitive dye di-4-ANNEPS

The voltage-sensitive dye is used with success for MAP recording from the heart surface by optical way via fluorescence measurement. Common fluorescence method is based on use of di-4-ANEPPS. VSD undergoes changes in its electronic structure, and consequently its fluorescence spectra, in response to changes in the surrounding electric field. As the VSD emission spectra shifts with the electric field, MAP can be represented by measurement in a specific wavelength band. Another method of fluorescence measurement is ratiometric and takes the advantage of the emission spectra shift depending on cell membrane potential.

\subsection{Recording setup}

There are two different setups for MAP measurement used in this project, simple optical setup [2] and new advanced ratiometric setup.

Simple optical setup, presented on Figure 2, includes $150 \mathrm{~W}$ halogen light source with built-in IR filter, which prevents a preparation from heating and a band-pass filter $(560 \mathrm{~nm} \pm 30 \mathrm{~nm})$, which selects light at excitation maximum of the used dye. The light goes through bifurcated fiber cable, which ensures two different ways of light. In detail: source light leads to the heart surface and the emitted light from heart to optical sensor. The emitted light is converted to an electric signal by a photodiode detector and amplified by a two-stage operational amplifier. Intensity of the detected light corresponds with MAP.

The data acquisition card digitized the preamplified MAP signals with 16 bits dynamic range and at rate of 2000 samples per second. The signals were acquired by a LabView compatible data acquisition multifunction card PCI-6250 (National Instruments, USA).

The main drawback of the method based on simple optical setup is large motion artefact present in MAP recordings caused by relative movement of optical probe with the heart surface. Optical probe, which is the end of bifurcated fiber cable, is passing through bath wall to the heart surface. However, the heart cannot be properly mechanically fixed while the fiber cable tip is only in touch with the heart surface. One of the solutions avoiding chemically induced electro-mechanical uncoupling is ratiometric recording.

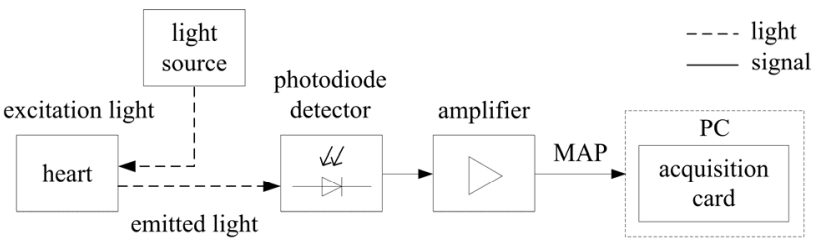

Figure 2. Block diagram of the simple recording setup.

Ratiometric recording setup is based on measurements at two different wavelengths or two narrow wavelength bands located left and right from the centre of emission spectrum and two signals corresponding to MAP are recorded. Thus, shift of emission spectra showed in Figure 1 is exploited to suppress noisy parts of the recorded signals. The idea is that one of the recorded signals is directly proportional to the MAP and the other is indirectly proportional.

In common ratiometric acquisition systems [3], the emitted light is split into two beams by a dichroic mirror (see Figure 3). Green light beam is detected by a green light sensitive photodiode and converted to an electrical signal - it is labeled as $\mathrm{MAP}_{\mathrm{G}}$ on Figure 3. Analogically red light beam is separately detected by red light sensitive photodiode and converted to a signal denoted as $\mathrm{MAP}_{\mathrm{R}}$. Then, MAP signal is computed as a ratio of these two auxiliary signals. Motion artefacts presented in both optical signals are divided and thus suppressed.

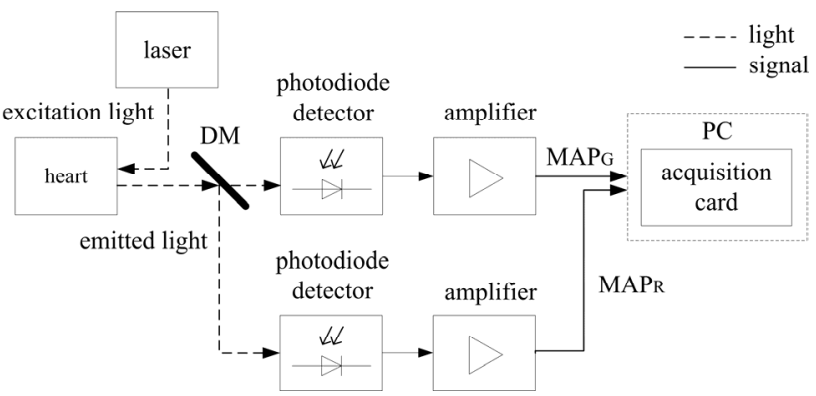

Figure 3. Block diagram of the two-photodiode ratiometric recording setup with a beam splitter. DM dichroic mirror, $\mathrm{MAP}_{\mathrm{G}}-$ green light beam converted by a photodiode to an electric signal, $\mathrm{MAP}_{\mathrm{R}}$ - red light beam converted by a photodiode to an electric signal.

Disadvantage of the published system might be loss of light which goes through a dichroic mirror. This can be compensated by using of more photodiodes in one package sensitive to more spectral bands.

The proposed ratiometric acquisition system includes a package of two photodiodes sensitive to green and red 
visible light (its block diagram is shown on Figure 5). Sensitive areas of the photodiodes is optimally placed into emitted light beam. Spectral responses of the used photodiodes can be seen on Figure 4.

Thus, a beam splitter which attenuates the emitted light is replaced. As a source of excitation light, blue laser is used with wavelength of $473 \mathrm{~nm}$ and controlled output power 0 to $20 \mathrm{~mW}$. Excitation light wavelength meets band of excitation for used VSD and also is outward narrow bands of photodiodes spectral responses.

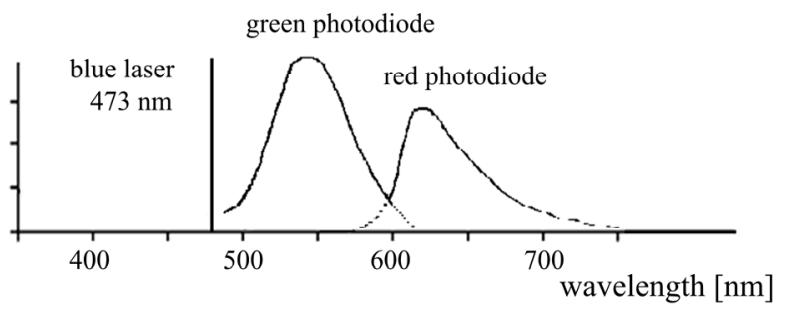

Figure 4. Spectral responses of green and red photodiodes provided a blue laser is used for excitation.

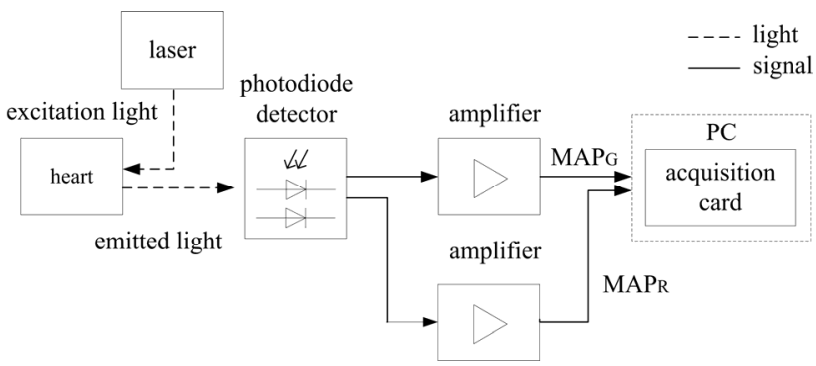

Figure 5. Block diagram of the proposed two-photodiode ratiometric recording setup. $\mathrm{MAP}_{\mathrm{G}}-$ green light beam converted by a photodiode to an electric signal, $\mathrm{MAP}_{\mathrm{R}}-$ red light beam converted by a photodiode to an electric signal.

Both signals from output of photodiode detectors were separately preamplified by bioamplifiers DAM50 (World Precision Instruments, Inc.). Then, $\mathrm{MAP}_{\mathrm{G}}$ and $\mathrm{MAP}_{\mathrm{R}}$ are digitized by the data acquisition card with 16 bits dynamic range and at rate of 2000 samples per second. The signals were acquired by a LabView compatible data acquisition multifunction card PCI-6250 (National Instruments, USA). Thus, the acquisition parts of the simple optical setup and the proposed-photodiode ratiometric recording setup were same for possible comparison of their properties.

\section{Results}

Quality of MAP recordings should be evaluated by signal-to-noise ration (SNR), according to formula:

$\mathrm{SNR}=20 \times \log ($ signal $/$ noise $)[\mathrm{dB}]$ where value of the signal is defined as height of ascending edge of MAP in milivolts and noise value is defined as difference between maximum and minimum values of the noise signal in milivolts in signal period between two subsequent MAPs.

An example of MAP signal recorded by the simple optical setup is shown on Figure 6, SNR was estimated as $20 \mathrm{~dB}$.

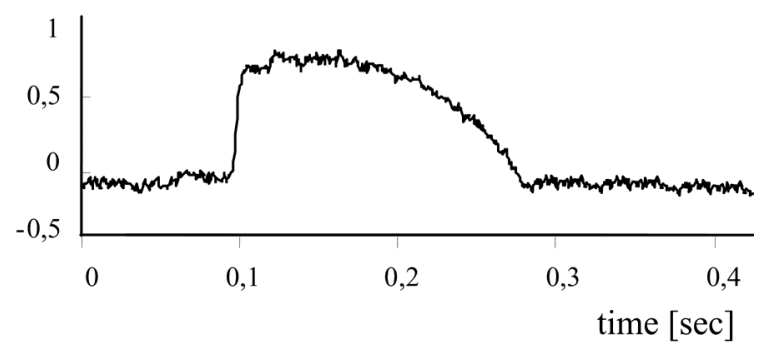

Figure 6. MAP signal recorded by the simple optical setup.

An example of MAP signal recorded by the proposed two-photodiode ratiometric recording setup is shown on Figure 7, SNR was estimated as 36dB.

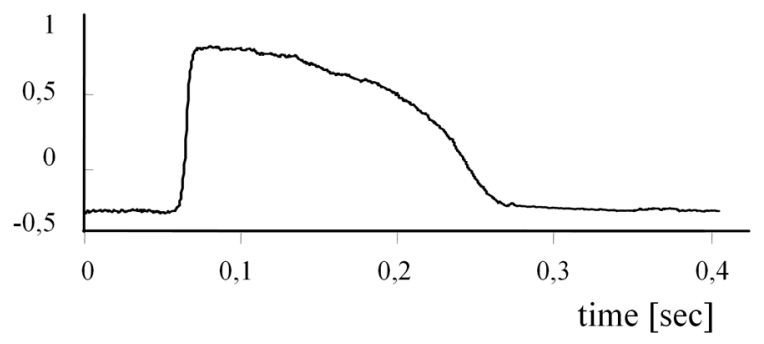

Figure 7. MAP signal recorded by the two-photodiode ratiometric recording setup.

Signal-to-noise ratio (SNR) was evaluated for each MAP signals recorded during six experiments. Three experiments were done for each compared recording setup.

The average SNR for the common ratiometric system was $\mathrm{SNR}=20 \mathrm{~dB}$ in average. The proposed system consistently allowed to get MAP signals with minimally $\mathrm{SNR}>30 \mathrm{~dB}$.

\section{Discussion and conclusions}

Monophasic action potentials can be efficiently recorded from the heart surface by optical methods via fluorescence measurement using voltage-sensitive dyes. Main drawbacks of these methods are motion artefacts present in the recorded signals due to unpredictable relative movement of optical probe with the heart surface and low signal-to-noise ratio caused by low intensity of 
emitted light and its attenuation by optical filters and splitters of the recording setup.

Two optical recording setups were designed and built. First, a simple optical setup consisting of a wide spectrum halogen lamp light source with optical filters to excite voltage-sensitive dye in a narrow spectral band, a single photodiode detector preceded with an optical filter to suppress the excitation light reflected from the heart tissue, an amplifier and an acquisition card for digitization of the recorded signal was built and tested. Such a recording setup is simple and provides a good quality signal which is slightly suppressed due to the use of optical filters. However, this system does not offer any possibility to suppress motion artefacts present in recorded MAPs.

Second, an optical recording setup with two photodiode sensor for ratiometric measurement was proposed and built as an alternative to existing systems that use a beam splitter. Ratiometric methods are efficient for suppression of motion artefacts. However, they require two sensors for recording in different spectral bands. Splitting an emission light beam into two ways attenuates light intensity in both channels and the resulting signals are more noisy comparing to the simple system. The proposed system does not require any beam splitter and exploits a package of two photodiode sensors sensitive in two different spectral bands. It only requires the use of a blue laser to avoid using optical filters. Such a system is efficient in suppression of motion artefacts and it also produces signals with higher signal-to-noise ratio comparing to published systems.

\section{Acknowledgements}

This work was supported by the grant projects of the Grant Agency GACR 102/07/1473, GACR 102/07/P521 and MSM 0021622402.

\section{References}

[1] Salama G. Optical mapping: background and historical perspective. In: Rosenbaum DS. and Jalife J. Optical Mapping of Cardiac Excitation and Arrhythmias 2001. Futura Publishing Company, Inc. Chapter 1:9-31.

[2] Provaznik I, Novakova M, Vesely Z, Blaha M, Chmelar M. Electro-Optical Recording System for Myocardial Ischemia Studies in Animal Experiments. In Computers in Cardiology. Danvers, USA: IEEE, 2003, pp.573-576.

[3] Knisley SB, Justice RK, Kong W, Johnson PL. Ratiometry of transmembrane voltage-sensitive fluorescent dye emission in hearts. Am J Physiol Heart Circ Physiol. 2000 Sep; 279(3):H1421-33

[4] Tai D C-S, Caldwell BJ, LeGrice IJ, Hooks DA, Pullan AJ, Smaill BH. Correction of Motion Artifact in Transmembrane Voltage-Sensitive Fluorescent Dye Emission in Hearts. Am J Physiol Heart Circ Physiol. 2004; 287:H985-H993

Address for correspondence

Jana Bardonova

Department of Biomedical Engineering

Kolejni 4

61200, Brno

Czech Republic

bardona@feec.vutbr.cz 